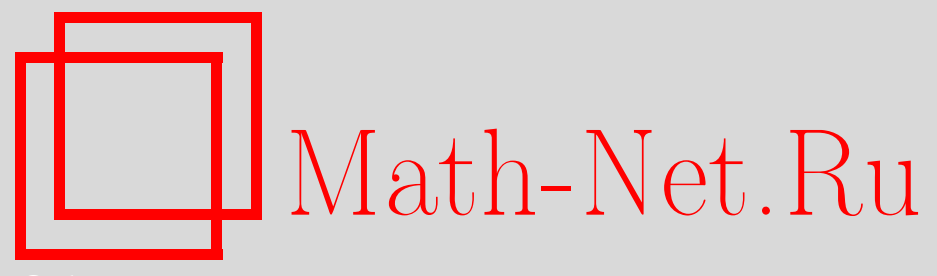

А. Н. Тимашёв, Случайные разбиения множеств с известным числом блоков, Дискрет. матем., 2003, том 15, выпуск $2,138-148$

DOI: https://doi.org/10.4213/dm201

Использование Общероссийского математического портала Math-Net.Ru подразумевает, что вы прочитали и согласны с пользовательским соглашением http://www . mathnet.ru/rus/agreement

Параметры загрузки:

IP : 18.209 .158 .208

26 апреля 2023 г., 13:56:35

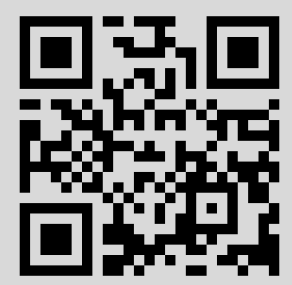




\title{
Случайные разбиения множеств с известным числом блоков
}

\author{
() 2003 г. А. Н. Тимашёв
}

\begin{abstract}
Рассматривается класс всех разбиений $n$-элементного множества на $N$ блоков. В предположении, что на этом классе задано равновероятное распределение и $n, N \rightarrow \infty$, получены асимптотики математического ожидания и дисперсии и доказаны пуассоновские и локальная нормальная теоремы для случайной величины, равной числу блоков заданного размера в случайно выбранном разбиении. Найдены асимптотические разложения для числа разбиений $n$-элементного множества на $N$ блоков, имеющих ровно $k=k(n, N)$ блоков фиксированного размера.
\end{abstract}

Рассмотрим класс всех разбиений $n$-элементного множества на $N$ попарно непересекающихся непустых подмножеств (блоков). Число таких разбиений равно $\sigma(n, N)$, где $\sigma(n, N)$ - числа Стирлинга второго рода, $N=1, \ldots, n[1]$. Пусть $k$ - целое неотрицательное число и $r$ - натуральное число (всюду далее, если не оговорено противное, $r$ фиксировано). Предполагая, что на этом классе задано равновероятное распределение, на соответствующем вероятностном пространстве определим случайную величину $\chi_{r}(n, N)$, равную числу блоков мощности (размера) $r$ в случайно выбранном разбиении. Если $B_{k r}(n, N)-$ число разбиений указанного вида, имеющих каждое ровно $k$ блоков размера $r$, то

$$
B_{k r}(n, N)=\sigma(n, N) \mathbf{P}\left\{\chi_{r}(n, N)=k\right\} .
$$

Пусть при $n, N \rightarrow \infty$ целое неотрицательное число $k=k(n, N)$ меняется так, что выполнены условия

$$
N-k \rightarrow \infty, \quad 1<\alpha_{0} \leqslant \alpha=\frac{n-k r}{N-k} \leqslant \alpha_{1}<\infty,
$$

где $\alpha_{0}, \alpha_{1}-$ постоянные и $\alpha_{0}>2$ при $r=1$.

Заметим, что при условиях (2) параметры $n, N$ меняются в центральной области в том смысле, что

$$
1<\beta_{0} \leqslant \beta=n / N \leqslant \beta_{1}<\infty,
$$

где $\beta_{0}, \beta_{1}-$ постоянные.

В работе получена асимптотика чисел $B_{k r}(n, N)$ и доказана локальная нормальная теорема, оценивающая вероятность $\mathbf{P}\left\{\chi_{r}(n, N)=k\right\}$ в предположении, что $n, N \rightarrow \infty$ и выполнены условия (2). Получены также достаточные условия сходимости распределения случайной величины $\chi_{r}(n, N)$ к пуассоновскому закону, справедливые при $n, N \rightarrow \infty$ в 
трех различных областях изменения параметров $n, N$. В доказательствах соответствующих теорем используются равенство (1) и известные оценки чисел Стирлинга второго рода $\sigma(n, N)$, а также метод условных распределений для сумм независимых случайных величин [2]. Заметим, что случайные разбиения множеств - сравнительно новое направление в комбинаторных исследованиях с использованием вероятностных методов (это отмечается в Главе 4 в [3]), и известные результаты в этой области носят менее законченный характер, чем, например, в схемах случайных размещений. В этом направлении укажем работы В. Н. Сачкова [1, 3-6], а также [7-9] (более подробную библиографию см. в [6]). Насколько известно автору, случайные разбиения конечных множеств с заданным числом блоков до настоящего времени систематически не изучались (за исключением так называемых противоречивых разбиений, см. обзор [6]).

Теорема 1. Пусть $n, N \rightarrow \infty$ и челое неотричательное число $k=k(n, N)$ меняется так, что выполнены условия (2). Тогда равномерно относительно $\alpha \in\left[\alpha_{0}, \alpha_{1}\right]$

$$
\begin{aligned}
B_{k r}=\frac{n !}{N !} \frac{\left(e^{\lambda_{r}}-1\right)^{N}}{\lambda_{r}^{n}}\left(\begin{array}{l}
N \\
k
\end{array}\right) & \left(\frac{p_{r}\left(\lambda_{r}\right)}{1-e^{-\lambda_{r}}}\right)^{k} \\
& \times\left(1-\frac{p_{r}\left(\lambda_{r}\right)}{1-e^{-\lambda_{r}}}\right)^{N-k} \frac{1}{\sigma_{r} \sqrt{2 \pi(N-k)}}(1+O(1 /(N-k))),
\end{aligned}
$$

где $\lambda=\lambda_{r}-$ единственный положительный корень уравнения

$$
\begin{aligned}
& \left(1-e^{-\lambda}-p_{r}(\lambda)\right)^{-1}\left(\lambda-r p_{r}(\lambda)\right)=\alpha, \\
& p_{r}(\lambda)=\frac{\lambda^{r}}{r !} e^{-\lambda}, \quad r=1,2 \ldots, \\
& \quad \sigma_{r}^{2}=\left(1-e^{-\lambda_{r}}-p_{r}\left(\lambda_{r}\right)\right)^{-1}\left(\lambda_{r}^{2}+\lambda_{r}-r^{2} p_{r}\left(\lambda_{r}\right)\right)-\alpha^{2}>0 .
\end{aligned}
$$

Доказательство. Нетрудно показать, что к рассматриваемой задаче применима обобщенная схема размещения (см. например, [10]), поэтому при $\lambda>0, r=1,2, \ldots$, справедливо представление

$$
\begin{aligned}
\mathbf{P}\left\{\chi_{r}(n, N)=k\right\}=\left(\begin{array}{l}
N \\
k
\end{array}\right)\left(\frac{p_{r}(\lambda)}{1-e^{\lambda}}\right)^{k}(1 & \left.-\frac{p_{r}(\lambda)}{1-e^{-\lambda}}\right)^{N-k} \\
& \times \frac{\mathbf{P}\left\{S_{N-k}^{(r)}=n-k r\right\}}{\mathbf{P}\left\{S_{N}=n\right\}}, \quad k=0,1, \ldots, N,
\end{aligned}
$$

где

$$
S_{N}=\xi_{1}+\ldots+\xi_{N}
$$

$\xi_{1}, \ldots, \xi_{N}-$ независимые в совокупности одинаково распределенные случайные величины, для которых

$$
\mathbf{P}\left\{\xi_{1}=m\right\}=\frac{p_{m}(\lambda)}{1-e^{-\lambda}}, \quad m=1,2, \ldots,
$$

наконец,

$$
S_{N-k}^{(r)}=\xi^{(r)}+\ldots+\xi_{N-k}^{(r)}
$$


$\xi_{1}^{(r)}, \ldots, \xi_{N-k}^{(r)}$ - независимые в совокупности одинаково распределенные случайные величины такие, что

$$
\begin{aligned}
& \mathbf{P}\left\{\xi_{1}^{(r)}=m\right\}=\mathbf{P}\left\{\xi_{1}=m \mid \xi_{1} \neq r\right\}=\frac{p_{m}(\lambda)}{1-e^{-\lambda}-p_{r}(\lambda)}, \quad m=1,2, \ldots, \quad m \neq \neq r, \\
& \mathbf{P}\left\{\xi_{1}^{(r)}=r\right\}=0 .
\end{aligned}
$$

Из этих условий следует, что

$$
\mathbf{P}\left\{S_{N}=n\right\}=\frac{N ! \lambda^{n}}{n !\left(e^{\lambda}-1\right)^{N}} \sigma(n, N),
$$

поскольку

$$
\sigma(n, N)=\frac{n !}{N !} \sum_{k_{1}+\ldots+k_{N}=n} \frac{1}{k_{1} ! \ldots k_{N} !}
$$

Подставляя (9) в (8) и используя (1), находим, что

$$
B_{k r}(n, N)=\frac{n !}{N !} \frac{\left(e_{\lambda}-1\right)^{N}}{\lambda^{n}}\left(\begin{array}{l}
N \\
k
\end{array}\right)\left(\frac{p_{r}(\lambda)}{1-e^{-\lambda}}\right)^{k}\left(1-\frac{p_{r}(\lambda)}{1-e^{-\lambda}}\right)^{N-k} \mathbf{P}\left\{S_{N-k}^{(r)}=n-k r\right\}
$$

Нетрудно подсчитать, что

$$
\mathbf{E} \xi_{1}^{(r)}=\left(1-e^{-\lambda}-p_{r}(\lambda)\right)^{-1}\left(\lambda-r p_{r}(\lambda)\right), \quad r=1,2, \ldots
$$

Если потребовать, чтобы $\mathbf{E} \xi_{1}^{(r)}=\alpha$, то окажется, что

$$
\mathbf{E} S_{N-k}^{(r)}=(N-k) \mathbf{E} \xi_{1}^{(r)}=n-k r
$$

и параметр $\lambda>0$ удовлетворяет уравнению (5). Легко проверить, что при $\alpha>1$ и $r \geqslant 2$ уравнение (5) имеет единственный положительный корень $\lambda=\lambda_{r}$ (при $r=1$ это верно, если $\alpha>2$ ). Таким образом, при условиях (2) это уравнение имеет ровно один положительный корень $\lambda=\lambda_{r}$.

Вычислим дисперсию $D \xi_{1}^{(r)}$. Нетрудно убедиться в том, что при $\lambda=\lambda_{r}$

$$
\mathbf{D} \xi_{1}^{(r)}=\sigma_{r}^{2}
$$

где значение $\sigma_{r}^{2}$ определяется согласно (6), (7). При этом $\sigma_{r}^{2}>0$, поскольку при наших условиях распределение случайной величины $\xi_{1}^{(r)}$ невырожденное. Применяя к оценке вероятности

$$
\mathbf{P}\left\{S_{N-k}^{(r)}=n-k r\right\}=\mathbf{P}\left\{S_{N-k}^{(r)}=\mathbf{E} S_{N-k}^{(r)}\right\}
$$

метод перевала (см. ниже (18), (19) и теорему 2), убеждаемся, что

$$
\mathbf{P}\left\{S_{N-k}^{(r)}=n-k r\right\}=\frac{1}{\sigma_{r} \sqrt{2 \pi(N-k)}}(1+O(1 /(N-k)))
$$

равномерно относительно $\alpha \in\left[\alpha_{0}, \alpha_{1}\right]$.

Полагая в (10) $\lambda=\lambda_{r}$ и используя (11), получаем оценку (4). Теорема 1 доказана. 
Формула (4) позволяет при выполнении условий теоремы 1 оценить число разбиений $B_{k r}(n, N)$ множества из $n$ элементов на $N$ блоков, каждое из которых имеет ровно $k$ блоков размера $r$. Отметим, что при $k=0$ и $r=1$ числа $B_{01}(n, N)$ есть так называемые присоединенные числа Стирлинга второго рода [12]. В этом случае из (4) получаем следующее утверждение.

Следствие 1. Пусть $n, N \rightarrow \infty$ так, что выполнены условия (3), причем $\beta_{0}>2$. Тогда равномерно относительно $\beta \in\left[\beta_{0}, \beta_{1}\right]$

$$
B_{01}(n, N)=\frac{n !}{N !} \lambda_{1}^{-n}\left(e^{\lambda_{1}}-1-\lambda_{1}\right)^{N} \frac{1}{\sigma_{1} \sqrt{2 \pi N}}\left(1+O\left(N^{-1}\right)\right)
$$

где $\lambda=\lambda_{1}-$ единственный положительный корень уравнения (5) и величина $\sigma_{1}^{2}>0$ определяется из (7) при $r=1 u \alpha=\beta$.

Оценка (12) дает хорошее приближение даже при небольших значениях $n$ и $N$. Так, например, если $n=10, N=4, r=1, k=0$, то $\beta=5 / 2$ и $\lambda_{1}=1,229934 \ldots$ с точностью до $10^{-6}$. В этом случае оценка (12) дает значение 9934 (с точностью до целых), если отбросить член $O\left(N^{-1}\right)$, в то время как $B_{01}(10 ; 4)=9450$.

Чтобы уточнить формулы (4) и (11), оценим коэффициенты ряда Крамера-Эссеена, дающего асимптотическое разложение остаточного члена вида $O(1 /(N-k))$ в этих формулах. Поскольку $\mathrm{E} S_{N-k}^{(r)}=n-k r$ при $\lambda=\lambda_{r}$, такие коэффищиенты могут быть отличны от нуля только при целых отрицательных степенях вида $(N-k)^{-m}, m=1,2, \ldots$ Для простоты ограничимся оценкой коэффициента $A_{1}$ при $(N-k)^{-1}$ в случае $m=1$. Известно [13], что

$$
A_{1}=\frac{1}{8} \varkappa_{4}^{(r)} \sigma_{r}^{-4}-\frac{5}{24}\left(\varkappa_{3}^{(r)}\right)^{2} \sigma_{r}^{-6}
$$

где $\varkappa_{s}^{(r)}-$ кумулянт порядка $s$ случайной величины $\xi_{1}^{(r)}, s=3,4$.

Нетрудно подсчитать, что

$$
\begin{aligned}
\varkappa_{3}^{(r)} & =\mathbf{E}\left(\xi_{1}^{(r)}-\mathbf{E} \xi^{(r)}\right)^{3}=m_{3}-3 \alpha \sigma_{r}^{2}-\alpha^{3}, \\
m_{3} & =\mathbf{E}\left(\xi_{1}^{(r)}\right)^{3}=\frac{\lambda_{r}^{3}+3 \lambda_{r}^{2}+\lambda_{r}-r^{3} p_{r}\left(\lambda_{r}\right)}{1-e^{-\lambda_{r}}-p_{r}\left(\lambda_{r}\right)}, \\
\varkappa_{4}^{(r)} & =\mathbf{E}\left(\xi_{1}^{(r)}-\mathbf{E} \xi_{1}^{(r)}\right)^{4}-3 \sigma_{r}^{4}=m_{4}-4 \alpha m_{3}+6 \alpha^{2} \sigma_{r}^{2}+3 \alpha^{4}-3 \sigma_{r}^{4}, \\
m_{4} & =\mathbf{E}\left(\xi_{1}^{(r)}\right)^{4}=\frac{\lambda_{r}^{4}+6 \lambda_{r}^{3}+7 \lambda_{r}^{2}+\lambda_{r}-r^{4} p_{r}\left(\lambda_{r}\right)}{1-e^{-\lambda_{r}}-p_{r}\left(\lambda_{r}\right)}
\end{aligned}
$$

Таким образом, имеет место следующее утверждение.

Следствие 2. При условиях теоремы 1 равномерно относительно $\alpha \in\left[\alpha_{0}, \alpha_{1}\right]$ справедлива оченка (4) с заменой остаточного члена вида $O(1 /(N-k))$ на $A_{1}(N-k)^{-1}+O\left((N-k)^{-2}\right)$, где значение $A_{1}$ определяется согласно (13)-(17).

Утверждение следствия 2 дает гораздо более точную оценку чисел $B_{k r}(n, N)$ по сравнению с формулой (4). Так, в примере, рассмотренном выше, нетрудно подсчитать, что $A_{1}=-0,197113 \ldots$ с точностью до $10^{-6}$, и поэтому соответствующая оценка с отбрасыванием остаточного члена вида $O\left(N^{-2}\right)$ дает значение 9445 с относительной погрешностью порядка $-0,00055$. 
Аналогичные (13), но более сложные формулы для коэффициентов $A_{m}$ при $(N-k)^{-m}$ в асимптотическом разложении остаточного члена вида $O\left((N-k)^{-1}\right)$ в (4) и (11) можно выписать и при $m \geqslant 2$.

Другой способ определения коэффициентов $A_{m}$ основан на применении метода перевала и исходит из легко проверяемого равенства

$$
\mathbf{P}\left\{S_{N-k}^{(r)}=n-k r\right\}=\frac{1}{2 \pi i} \oint \frac{(P(z))^{N-k}}{z^{n-k r+1}} d z,
$$

где

$$
P(z)=\frac{e^{\lambda_{r}(z-1)}-e^{-\lambda_{r}}-z^{r} p_{r}\left(\lambda_{r}\right)}{1-e^{-\lambda_{r}}-p_{r}\left(\lambda_{r}\right)}
$$

и интегрирование осуществляется по окружности с центром в нуле, пробегаемой в положительном направлении.

Уравнение точки перевала $z P^{\prime}(z) / P(z)=\alpha$ с учетом (5) и (19) дает единственное значение корня $z=1$, лежащее на вещественной положительной полуоси. При этом

$$
P(1)=1, \quad P^{\prime}(1)=\alpha, \quad P^{\prime \prime}(1)=\sigma_{r}^{2}+\alpha(\alpha-1) .
$$

Учитывая эти равенства и используя стандартную технику метода перевала [14], нетрудно вывести из (18) и (19), что справедлива следующая теорема.

Теорема 2. При условиях теоремы 1 остаточный член вида $O\left((N-k)^{-1}\right)$ в (4) можно заменить на ряд

$$
\sum_{m=1}^{\infty} A_{m}(N-k)^{-m},
$$

если знак равенства заменить символом асимптотического разложения, справедливого равномерно относительно $\alpha \in\left[\alpha_{0}, \alpha_{1}\right]$. Коэффичиенты $A_{m}$ этого разложения определяются из соотношений

$$
A_{m}=-\frac{i \sigma_{r}(2 m) !}{m ! 2^{2 m+1 / 2}} a_{2 m}, \quad m=1,2, \ldots,
$$

где

$$
\sum_{s=0}^{\infty} a_{s} u^{s}=\eta^{\prime}(u)(\eta(u))^{-1}
$$

при всех достаточно малых по модулю значениях и,

$$
a_{0}=i \sqrt{2} \sigma_{r}^{-1}
$$

и функция $\eta($ (и) является аналитическим в окрестности нуля решением дифференциального уравнения

$$
\eta^{\prime}(u)\left(P^{\prime}(\eta(u))(P(\eta(u)))^{-1}-\alpha(\eta(u))^{-1}\right)+2 u=0
$$

удовлетворяючим начальному условию $\eta(0)=1$, значение $P$ определяется из (19). 
Нетрудно проверить, что коэффициенты $A_{m}$ в теореме 2 определены так, что каждый из них является непрерывной функщией параметра $\alpha$ (и поэтому ограничен на отрезке $\left[\alpha_{0}, \alpha_{1}\right]$. Отсюда следует, что соответствующее асимптотическое разложение справедливо равномерно относительно $\alpha \in\left[\alpha_{1}, \alpha_{2}\right]$. На уровне главного члена асимптотики интегралов вида (18) этот факт отмечен в [18].

Теорема 3. При условиях теоремы 1 равномерно относительно $\alpha \in\left[\alpha_{0}, \alpha_{1}\right]$ и чельх положительных значений

$$
k=N q_{r}\left(\lambda_{r}\right)+x \sqrt{N},
$$

для которых $q_{r}(\lambda)=\left(p_{r}(\lambda)\right) /\left(1-e^{-\lambda}\right)$ и величина $x$ заключена в любом фиксированном конечном интервале,

$$
\begin{aligned}
& \mathbf{E}_{\chi_{r}}(n, N)=N q_{r}\left(\lambda_{r}\right)(1+O(1 /(\sqrt{N}))), \\
& \mathbf{D} \chi_{r}(n, N)=N\left(\sigma^{(r)}\right)^{2}(1+O(1 /(\sqrt{N})))
\end{aligned}
$$

2de

$$
\begin{aligned}
\left(\sigma^{(r)}\right)^{2} & =\frac{\sigma_{r}^{2}}{\sigma^{2}}\left(1-q_{r}\left(\lambda_{r}\right)\right)^{2} q_{r}\left(\lambda_{r}\right)>0, \\
\sigma^{2} & =\left(1-e^{-\lambda_{r}}\right)^{-2}\left(1-\left(1+\lambda_{r}\right) e^{-\lambda_{r}}\right) \lambda_{r}>0 .
\end{aligned}
$$

Доказательство. Нетрудно подсчитать, что в обозначениях (8) и (21) $\mathbf{D} \xi_{1}=\sigma^{2}$.

Применяя для оценки вероятности $\mathbf{P}\left\{S_{N}=n\right\}$ метод перевала и учитывая равенство

$$
M=\mathbf{E} \xi_{1}=\left(1-e^{-\lambda_{r}}\right)^{-1} \lambda_{r}
$$

и равенство

$$
(n-M N) / \sqrt{N}=(r-\alpha) x,
$$

которое следует из (5), (22) и условий теоремы 3 , получаем, что

$$
\left.\mathbf{P}\left\{S_{N}=n\right\}=\frac{1}{\sqrt{2 \pi N} \sigma} \exp \left(-\frac{(r-\alpha)^{2} x^{2}}{2 \sigma^{2}}\right)\right)(1+O(1 / \sqrt{N})) .
$$

Следовательно, согласно (9)

$$
\sigma(n, N)=\frac{n !}{N !} \lambda_{r}^{-n}\left(e^{\lambda_{r}}-1\right)^{N} \frac{1}{\sqrt{2 \pi N} \sigma} \exp \left(-\frac{(r-\alpha)^{2} x^{2}}{2 \sigma^{2}}\right)(1+O(1 / \sqrt{N})) .
$$

При замене $n$ на $n-r, N$ на $N-1$ и $k$ на $k-1$ равенство $\alpha=(n-k r) /(N-k)$, справедливое согласно (2), сохраняется, поэтому из условий теоремы 3 получаем, что

$$
\frac{\sigma(n-r, N-1)}{\sigma(n, N)}=\frac{(n-r) ! N}{n !} \lambda_{r}^{r}\left(e^{\lambda_{r}}-1\right)^{-1}(1+O(1 / \sqrt{N})) .
$$

С помощью несложных комбинаторных рассуждений нетрудно убедиться в том, что факториальные моменты случайной величины $\chi_{r}(n, N)$ определяются соотношениями

$$
\mathbf{E}\left(\chi_{r}(n, N)\right)_{j}=\frac{n !}{(n-r j) !(r !)^{j}} \frac{\sigma(n-r j, N-j)}{\sigma(n, N)}, \quad j=1,2, \ldots
$$


В частности, при $j=1$

$$
\mathbf{E}_{\chi_{r}}(n, N)=\left(\begin{array}{l}
n \\
r
\end{array}\right) \frac{\sigma(n-r, N-1)}{\sigma(n, N)}=N q_{r}\left(\lambda_{r}\right)(1+O(1 / \sqrt{N})) .
$$

При $j=2$ получаем, что

$$
\mathbf{E}\left(\chi_{r}(n, N)\right)^{2}=\mathbf{E}_{\chi_{r}}(n, N)+\frac{n !}{(n-2 r) !(r !)^{2}} \frac{\sigma(n-2 r, N-2)}{\sigma(n, N)} .
$$

Используя эту формулу и соображения, аналогичные приведенным выше, можно вывести асимттотическую оценку для дисперсии $\mathrm{D}_{\chi_{k}}(n, N)$; соответствующие (существенно более громоздкие) выкладки мы опускаем.

Теорема 3 доказана.

Теорема 4. При условиях теоремы 3 равномерно относительно $\alpha \in\left[\alpha_{0}, \alpha_{1}\right]$ и чельх положительных значений $k=N q_{r}\left(\lambda_{r}\right)+x \sqrt{N}$, для которых $|x| \leqslant C$, где C- положительная постоянная,

$$
\begin{aligned}
\mathbf{P}\left\{\chi_{r}(n, N)=k\right\} & =\frac{1}{\sqrt{2 \pi N\left(\sigma^{(r)}\right)^{2}}} \\
& \times \exp \left(-\frac{x^{2}}{2}\left(\frac{1}{q_{r}\left(\lambda_{r}\right)\left(1-q_{r}\left(\lambda_{r}\right)\right)}-\frac{(r-\alpha)^{2}}{\sigma^{2}}\right)\right)(1+O(1 / \sqrt{N})),
\end{aligned}
$$

где значения $\left(\sigma^{(r)}\right)^{2}$ и $\sigma^{2}$ определяются согласно (20) $u(21)$.

Доказательство. Оценка (25) следует из (8), (11) и (23), если учесть, что при условиях теоремы 4 по локальной теореме Муавра-Лапласа

$$
\begin{aligned}
& \left(\begin{array}{l}
N \\
k
\end{array}\right)\left(q_{r}\left(\lambda_{r}\right)\right)^{k}\left(1-q_{r}\left(\lambda_{r}\right)\right)^{N-k} \\
& \quad=\frac{1}{\sqrt{2 \pi N q_{r}\left(\lambda_{r}\right)\left(1-q_{r}\left(\lambda_{r}\right)\right)}} \exp \left(-\frac{x^{2}}{2 q_{r}\left(\lambda_{r}\right)\left(1-q_{r}\left(\lambda_{r}\right)\right)}\right)(1+O(1 / \sqrt{N}))
\end{aligned}
$$

и согласно (11)

$$
\mathbf{P}\left\{S_{N-k}^{(r)}=n-k r\right\}=\frac{1}{\sigma_{r} \sqrt{2 \pi N\left(1-q_{r}\left(\lambda_{r}\right)\right)}}(1+O(1 / \sqrt{N}))
$$

равномерно относительно $\alpha \in\left[\alpha_{0}, \alpha_{1}\right]$ и $x \in[-C, C]$, для которых $k \in \mathbf{N}$.

Теорема 4 доказана.

Предположим теперь, что $n, N \rightarrow \infty$ так, что выполнено условие (3). Если отказаться от ограничения, что $r$ фиксировано, то окажется, что справедлива следующая теорема.

Теорема 5. Пусть $n, N, r \rightarrow \infty$ так, что выполнено (3) $u N q_{r}\left(z_{0}\right) \rightarrow \lambda>0$, где $z=z_{0}$ - единственный положительный корень уравнения

$$
z\left(1-e^{-z}\right)^{-1}=\beta
$$

Тогда случайная величина $\chi_{r}(n, N)$ распределена в пределе по закону Пуассона с параметром $\lambda$. 
Доказательство. Известно [16], что если $n, N \rightarrow \infty$ так, что выполнено (3), то равномерно относительно $\beta \in\left[\beta_{0}, \beta_{1}\right]$

$$
\sigma(n, N)=\frac{n !}{N !} \frac{z_{0}^{N-n}}{\left(\beta-z_{0}\right)^{N} \sqrt{2 \pi n\left(1-\beta+z_{0}\right)}}(1+O(1 / N)) .
$$

Поэтому при условиях теоремы 5 для любого фиксированного $j=1,2, \ldots$

$$
\sigma(n-r j, N-j)=\frac{(n-r j) !}{(N-j) !} \frac{z_{1}^{N-n+r j}\left(e^{z_{1}}-1\right)^{-j}}{\left(\alpha-z_{1}\right)^{N} \sqrt{2 \pi(n-r j)\left(1-\alpha+z_{1}\right)}}(1+O(1 / N)),
$$

где $z=z_{1}$ - единственный положительный корень уравнения (26) с заменой $\beta$ на $\alpha=(n-r j) / N$. Но при наших условиях $r \sim \ln N / \ln \ln N$, и поэтому из (27) и (28) следует, что

$$
\frac{\sigma(n-r j, N-j)}{\sigma(n, N)}=\frac{(n-r j) ! N !}{n !(N-j) !} \frac{z_{0}^{r j}}{\left(e^{z_{0}}-1\right)^{j}}(1+o(1)) .
$$

Действительно, согласно (3) $\beta=\alpha+r j / N$, и нетрудно проверить, что

$$
\begin{aligned}
\frac{z_{1}}{z_{0}} & =1-\frac{r j}{\beta\left(1-\beta+z_{0}\right) N}+O\left(1 / N^{2}\right), \\
\left(\frac{z_{1}}{z_{0}}\right)^{N-n} & =\exp \left(-\frac{r j(1-\beta)}{\beta\left(1-\beta+z_{0}\right)}\right)(1+o(1)), \\
\left(\frac{\beta-z_{0}}{\alpha-z_{1}}\right)^{N} & =\exp \left(\frac{r j(1-\beta)}{\beta\left(1-\beta+z_{0}\right)}\right)(1+o(1)) .
\end{aligned}
$$

Из этих оценок и формул (27), (28) следует (29). Но тогда согласно (24) получаем, что при условиях теоремы 5 для любого фиксированного $j=1,2, \ldots$

$$
\mathbf{E}\left(\chi_{r}(n, N)\right)_{j}=N^{j}\left(q_{r}\left(z_{0}\right)\right)^{j}(1+o(1))=\lambda^{j}(1+o(1)) .
$$

Теорема 5 доказана.

Замечание 1. Теорему 5 можно также доказать следующим способом. Если воспользоваться пуассоновским приближением для биномиального распределения, то окажется, что при условиях этой теоремы для любого фиксированного $k=0,1, \ldots$

$$
\left(\begin{array}{l}
N \\
k
\end{array}\right)\left(q_{r}\left(z_{0}\right)\right)^{k}\left(1-q_{r}\left(z_{0}\right)\right)^{N-k}=\frac{\lambda^{k}}{k !} e^{-\lambda}(1+o(1)) .
$$

Таким образом, для доказательства теоремы 5 достаточно убедиться в том, что

$$
\mathbf{P}\left\{S_{N-k}^{(r)}=n-k r\right\}=\mathbf{P}\left\{S_{N}=n\right\}(1+o(1)) .
$$

Исходя из (3) и используя метод перевала для оценки вероятностей $\mathbf{P}\left\{S_{N-k}^{(r)}=n-k r\right\}$ и $\mathbf{P}\left\{S_{N}=n\right\}$, несложно проверить справедливость оценки (30) (а, тем самым, и утверждение теоремы 5).

Теорема 6. Пусть $n, N \rightarrow \infty$ так, что $N=o(n)$ u $N p_{r}(n / N) \rightarrow \lambda>0$. Тогда при любом фиксированном $r=1,2, \ldots$ справедливо утверждение теоремы 5. 
Доказательство. Известно [15], что если $n, N \rightarrow \infty$ так, что $N=o(n)$, то

$$
\sigma(n, N)=\frac{N^{n}}{N !} \exp \left(-N e^{-n / N}\right)(1+o(1))
$$

Согласно (24) достаточно убедиться в том, что для любого фиксированного $j=1,2, \ldots$

$$
\frac{\sigma(n-r j, N-j)}{\sigma(n, N)}=\lambda^{j}(r !)^{j} n^{-r j}(1+o(1)) .
$$

При наших условиях $n \sim N \ln N$, так что согласно (31) для любых фиксированных $r, j=1,2, \ldots$

$$
\sigma(n-r j, N-j)=\frac{(N-j)^{n-r j}}{(N-j) !} \exp \left(-(N-j) e^{-(n-r j) /(N-j)}\right)(1+o(1)) .
$$

Далее, справедливы оценки

$$
\frac{(N-j)^{n-r j} N !}{N^{n}(N-j) !}=\frac{N^{j}(r !)^{j}}{n^{r j}}\left(p_{r}(\beta)\right)^{j}(1+o(1))
$$

где $r, j=1,2, \ldots, \beta=n / N \sim \ln N, p_{r}(\beta)=\beta^{r} e^{-\beta} / r !$.

Следовательно, с учетом (31) и (33) для обоснования (32) достаточно проверить, что для любых фиксированных $r, j=1,2, \ldots$

$$
N e^{-n / N}=(N-j) e^{-(n-r j) /(N-j)}+o(1) .
$$

Так как

$$
j \exp (-(n-r j) /(N-j))=o(1)
$$

достаточно убедиться в том, что для любых фиксированных $r, j=1,2, \ldots$

$$
\varepsilon_{N}=N\left(e^{-n / N}-e^{-(n-r j) /(N-j)}\right)=o(1) .
$$

Но $n \sim N \ln N$, поэтому

$$
\varepsilon_{N}=N e^{-n / N}(1-\exp (-j \ln N / N+o(\ln N / N))) \sim j \ln N N^{-1+o(1)},
$$

откуда следует (34).

Теорема 6 доказана.

Теорема 7. Пусть $n, N \rightarrow \infty$ так, что при некотором фиксированном $r=3,4, \ldots$

$$
N(n / N-1)^{r-1} \rightarrow r ! \lambda / 2^{r-1},
$$

где $\lambda$ - положительная постоянная. Тогда случайная величина $\chi_{r}(n, N)$ распределена в пределе по закону Пуассона с параметром $\lambda$. 
Идея доказательства теоремы 7 состоит в следующем. Если $\beta=n / N$ и $z=z_{0}$ единственный положительный корень уравнения (26), то можно показать, что оценка (29) остается справедливой при $r \geqslant 3$ и условии (35), если остаточный член вида $O\left(N^{-1}\right)$ заменить на $o$ (1) (это верно и в том случае, когда $n, N \rightarrow \infty$ так, что $n-N=o(\sqrt{n}) \rightarrow \infty$; соответствующая оценка чисел Стирлинга второго рода $\sigma(n, N)$ содержится в [17]). Остается заметить, что из условия (35) и уравнения (26) следует, что

$$
\beta=1+o(1), \quad z_{0}=2(\beta-1)(1+o(1))=o(1),
$$

поэтому

$$
N z_{0}^{r}\left(e^{z_{0}}-1\right)^{-1} \sim N z_{0}^{r-1} \sim N 2^{r-1}(\beta-1)^{r-1}=r ! \lambda(1+o(1))
$$

и из (24) и (29) выводим, что для любого фиксированного $j=1,2, \ldots$

$$
\mathbf{E}\left(\chi_{r}(n, N)\right)_{j}=\lambda^{j}(1+o(1))
$$

Замечание 2. При $r=2$ условие (35) означает, что $n-N=\lambda \in \mathbf{N}$ при всех достаточно больших значениях $n$ и $N$. В этом случае согласно [17]

$$
\sigma(n, N)=\frac{n^{2 \lambda}}{2^{\lambda} \lambda !}(1+o(1))
$$

поэтому

$$
\mathbf{E}\left(\chi_{2}(n, N)\right)_{j}=\lambda(\lambda-1) \ldots(\lambda-j+1)(1+o(1)), \quad j=1, \ldots, \lambda,
$$

и $\mathbf{E}\left(\chi_{2}(n, N)\right)_{j}=0$ при $j>\lambda$. Таким образом, при $r=2$ из (35) следует, что распределение случайной величины $\chi_{2}(n, N)$ асимптотически вырождено в точке $\lambda$.

Пусть $\theta(n, N)$ - случайная величина, равная размеру блока, содержащего фиксированный элемент, в случайном разбиения $n$-элементного множества на $N$ блоков.

Теорема 8. Пусть $n, N \rightarrow \infty$ так, что выполнены условия (3). Тогда для любого фиксированного $k=1,2, \ldots$

$$
\mathbf{P}\{\theta(n, N)=k\}=\frac{z_{0}^{k-1}}{(k-1) !} e^{-z_{0}}(1+o(1)),
$$

где величина $z_{0}$ определена в теореме 5.

Оценка (36) следует из (26) и (29), если положить в (29) $j=1$ и $r=k$ и воспользоваться очевидными равенствами

$$
\mathbf{P}\{\theta(n, N)=k\}=\frac{\left(\begin{array}{c}
n-1 \\
k-1
\end{array}\right) \sigma(n-k, N-1)}{\sigma(n, N)}, \quad k=1, \ldots, n
$$




\section{Список литературы}

1. Сачков В. Н., Комбинаторные методы дискретной математики. Наука, Москва, 1977.

2. Колчин В. Ф., Один класс предельных теорем для условных распределений. Лит. матем. сб. (1968) 8, №1, 53-63.

3. Сачков В. Н., Вероятностные методы в комбинаторном анализе. Наука, Москва, 1978.

4. Сачков В. Н., Случайные разбиения множеств с помеченными подмножествами. Матем. сб. (1973) 92, №3, 491-502.

5. Сачков В. Н., Случайные разбиения множеств. Теория вероятностей и ее применения (1974) 19, №1, 178-194.

6. Сачков В. Н., Случайные разбиения множеств. Матем. вопросы киберн. (1999) 8, 33-54.

7. Arratia R., Tavare S., Independent process approximations for random combinatiorial structures. $A d v$. Math. (1994) 104, 90-154.

8. Pittel B., Random set partitions: asymptotic of subset counts. J. Comb. Theory (1997) 79, 326-359.

9. Mutafchiev L., Limiting distributions for the number of distinct component sizes in relational structures. J. Comb. Theory (1997) 79, 1-35.

10. Kolchin A. V., Random partitions of a set and the generalised allocation scheme. In: Probabilistic Methods in Discrete Mathematics. VSP, Utrecht, 2002, pp. 215-218.

11. Колчин В. Ф., Севастьянов Б. А., Чистяков В. П., Случайные размещения. Наука, Москва, 1976.

12. Риордан Дж., Введение в комбинаторный анализ. ИЛ, Москва, 1962.

13. Петров В. В., Суммы независимых случайных величин. Наука, Москва, 1972.

14. Евграфов М. А., Асимптотические оченки и челые функции. Физматлит, Москва, 1962.

15. Медведев Ю. И., Ивченко Г. И., Асимптотические представления конечных разностей от степенной функции в произвольной точке. Теория вероятностей и ее применения (1965) 10, №1, 151-156.

16. Good I. J., An asymptotic formula for the differences of the powers at zero. Ann. Math. Statist. (1961) 32, 249-256.

17. Hsu L. C., Note on an asymptotic expansion of the $n$th difference of zero. Ann. Math. Statist. (1948) 19, №2, 273-277.

18. Федорюк М. В., Асимптотика. Интегралы и ряды. Наука, Москва, 1987.

Статья поступила 11.04.2002. 